\title{
BMJ \\ open \\ A manual physical therapy approach versus subacromial corticosteroid injection for treatment of shoulder impingement syndrome: a protocol for a randomised clinical trial
}

\author{
Daniel I Rhon, ${ }^{1}$ Robert E Boyles, ${ }^{2}$ Joshua A Cleland, ${ }^{3}$ David L Brown ${ }^{4}$
}

To cite: Rhon DI, Boyles RE, Cleland JA, et al. A manual physical therapy approach versus subacromial corticosteroid injection for treatment of shoulder impingement syndrome: a protocol for a randomised clinical trial. BMJ Open 2011:1:e000137. doi:10.1136/bmjopen-2011000137

- Prepublication history for this paper is available online. To view these files please visit the journal online (http:// bmjopen.bmj.com).

Received 12 April 2011 Accepted 3 June 2011

This final article is available for use under the terms of the Creative Commons Attribution Non-Commercial 2.0 Licence; see http://bmjopen.bmj.com

For numbered affiliations see end of article.

Correspondence to

Dr Daniel Rhon;

dirhon@gmail.com

\section{ABSTRACT}

Introduction: Corticosteroid injections (CSI) are a recommended and often-used first-line intervention for shoulder impingement syndrome (SIS) in primary care and orthopaedic settings. Manual physical therapy (MPT) offers a non-invasive approach with negligible risk for managing SIS. There is limited evidence to suggest significant long-term improvements in pain, strength and disability with the use of MPT, and there are conflicting reports from systematic reviews that question the long-term efficacy of CSI. Specifically, the primary objective is to compare the effect of CSI and MPT on pain and disability in subjects with SIS at 12 months.

Design: This pragmatic randomised clinical trial will be a mixed-model $2 \times 5$ factorial design. The independent variables are treatment (MPT and CSI) and time with five levels from baseline to 1 year. The primary dependent variable is the Shoulder Pain and Disability Index, and the secondary outcome measures are the Global Rating of Change and the Numeric Pain Rating Scale. For each ANOVA, the hypothesis of interest will be the two-way group-by-time interaction. Methods and analysis: The authors plan to recruit 104 participants meeting established impingement criteria. Following examination and enrolment, eligible participants will be randomly allocated to receive a pragmatic approach of either CSI or MPT. The MPT intervention will consist of six sessions, and the CSI intervention will consist of one to three sessions. All subjects will continue to receive usual care. Subjects will be followed for 12 months.

Dissemination and ethics: The protocol was approved by the Madigan Army Medical Center Institutional Review Board. The results may have an impact on clinical practice guidelines. This study was funded in part by the Orthopaedic Physical Therapy Products Grant through the American Academy of Orthopaedic Manual Physical Therapists.

Trial Registration: http://clinicaltrials.gov/ NCT01190891.

\section{ARTICLE SUMMARY}

Article focus

- Shoulder pain is a common symptom in patients seeking healthcare for musculoskeletal complaints.

- Corticosteroid injections (CSI) are a common first-line intervention for shoulder pain in primary care settings, but their long-term efficacy has not been established.

- The long-term efficacy of manual physical therapy and CSI will be evaluated and compared from baseline out to 1 year after enrollment.

Key messages

- Manual physical therapy has been shown to provide improvements in pain and function in patients with shoulder impingement but has not been directly compared with CSI.

- Understanding which interventions have better long-term outcomes may be instrumental in helping improve clinical practice guidelines for the management of shoulder impingement syndrome.

Strengths and limitations of this study

- This randomised controlled study will compare the effectiveness of a manual physical therapy approach to a corticosteroid injection in patients with shoulder impingement.

- This is a pragmatic study evaluating two interventions that are standard practice and have been shown to be effective for shoulder impingement.

- Even as a single blinded randomised clinical trial there is no true control group, and we cannot state whether true a cause-and-effect relationship exists.

- Owing to the pragmatic nature of the study, the intervention will not be standardised, which could make it difficult for clinicians to replicate.

- The lack of a gold standard with the diagnosis of shoulder impingement makes this population difficult to study. 


\section{INTRODUCTION}

Shoulder impingement is among the most common function-limiting disorders of the musculoskeletal system. ${ }^{1}{ }^{2}$ The point prevalence of shoulder symptoms has been reported to range from 20 to $33 \%,{ }^{3}$ and the incidence of shoulder complaints in the general population is increasing. ${ }^{4}$ Furthermore, several authors have reported low rates of perceived recovery (patient reports of 'being cured') for patients with a new episode of shoulder pain. ${ }^{4-7}$ Less than $25 \%$ of patients with a first episode of shoulder pain may recover and be symptomfree after 3 months. ${ }^{5}$ Recovery rates at 18 months have been reported only between $49 \%$ and $59 \%,{ }^{568}$ and $25 \%$ of patients with shoulder or neck pain experience at least one episode of recurrence within 12 months. ${ }^{9}$ These findings suggest that shoulder pain can be recurrent and frequently progresses to the chronic stage.

A comprehensive manual physical therapy (MPT) approach, including both thrust and non-thrust techniques and reinforcing mobility exercises, is a common intervention for patients presenting with a primary report of shoulder pain. ${ }^{1}{ }^{9-16}$ This comprehensive approach has shown improvement in shoulder symptoms with mobilisation and manipulation techniques targeted to the thoracic spine, cervicothoracic spine, rib cage and acromioclavicular joints, ${ }^{9}{ }^{12}$ in addition to the glenohumeral joint. A recent systematic review by Ho et al $^{17}$ stated there was no clear evidence to suggest additional benefits of manual therapy to other interventions for shoulder impingement, indicating the need for further high-quality research. That systematic review was conducted through January of 2007, excluding some more recent trials discussed below.

In a trial by Bergman et $a l,{ }^{10}$ patients with a primary report of shoulder pain were randomly assigned to receive usual medical care for their shoulder symptoms from their primary care physicians or usual care plus manipulative therapy (UMC+MT) directed at the cervicothoracic spine and rib cage for a maximum of six treatment sessions. The group that received manipulative therapy reported increased rates of 'full recovery', and improved disability out to 52 weeks. ${ }^{10}$ In a trial by Bang and Deyle, ${ }^{9}$ significant improvements in function and pain with the use of an MPT approach, consisting of manual techniques and reinforcing exercises, were seen out at 2 months. Two other studies compared the use of manual mobilisation techniques in addition to a comprehensive offering of various therapeutic modalities and exercise, and showed improved outcomes in the groups that received mobilisations in addition to their comprehensive programme. ${ }^{10} 18$ Several other studies have looked at a less pragmatic approach to manual therapy by breaking down the manual therapy approach into several subcomponents. ${ }^{18} 19$ No evidence for harm with using this intervention has been reported.

Corticosteroids injections are a common intervention for symptoms from shoulder impingement and have also been shown to be an effective treatment for shoulder pain. They are one of the most common procedures for the management of shoulder pain used by orthopaedists, rheumatologists and general practitioners, ${ }^{20}$ with $96 \%$ of clinicians in a recent survey stating that CSI is an efficacious treatment option for rotator cuff pathology. ${ }^{21}$ Conflicting evidence in the literature suggests their efficacy in the management of shoulder impingement is not well established. ${ }^{22-27}$ A recent meta-analysis by Arroll and Goodyear-Smith ${ }^{23}$ evaluating the efficacy of CSI for painful shoulder conditions (included rotator cuff tendonitis and adhesive capsulitis) concluded that subacromial injections are probably effective for improvement of rotator cuff tendonitis when compared with placebo or non-steroidal anti-inflammatory drugs, but that there is still insufficient evidence to determine the extent of their effectiveness. Five studies compared subacromial injection with placebo and showed significantly better results then placebo in all studies, ${ }^{28}$ but only three of those studies were specifically for shoulder impingement. The determination of effectiveness was based on dichotomising results into success and nonsuccess based on the terms that included 'responder,' 'decreased pain' and 'remission.'

A recent Cochrane review ${ }^{24}$ and a meta-analysis by Gaujoux-Viala et $a l^{26}$ indicated that steroid injections were not more effective than non-steroidal anti-inflammatory drugs in the treatment of shoulder and elbow tendonitis. Another review by Koester $e t a l^{27}$ found nine randomised clinical trials comparing subacromial corticosteroid injection with placebo in patients with rotator cuff disease and concluded that subacromial corticosteroid injection is not effective for the treatment of rotator cuff disease. It has been reported that the variable success with subacromial CSI may be due to inaccuracy of the clinician in terms of approach (posterior or anterolateral), ${ }^{29}$ tendon penetration ${ }^{30}$ and/or inconsistencies in dose. ${ }^{23}$

\section{Specific aim}

The aim was to determine if there is any clinically significant difference in outcomes of pain and disability between subjects with shoulder impingement syndrome that receive MPT compared with CSI at 1 year.

\section{TRIAL DESIGN AND METHODS}

The study will be a randomised clinical trial. The independent variables are treatment (MPT and CSI) and time with five levels from baseline out to 1 year. The primary dependent variable (DV) is the Shoulder Pain and Disability Index (SPADI). The secondary DVs are the Global Rating of Change (GRC) and the Numeric Pain-Rating Scale (NPRS). Figure 1 demonstrates the flow of subjects through the trial. This will be a trial assessing pragmatic delivery of two common interventions, and will report results following CONSORT guidelines for pragmatic trials. ${ }^{31}$ The current SPIRIT guidelines for creating protocols for randomised clinical trials were followed. ${ }^{32}$ 


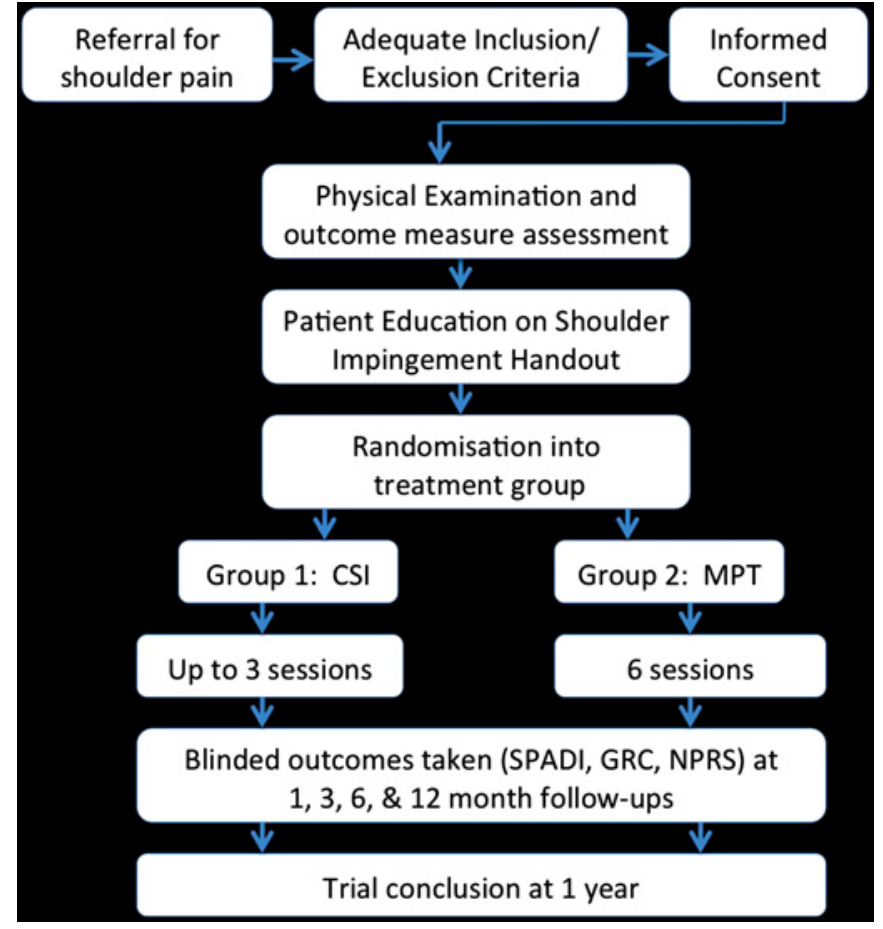

Figure 1 Proposed recruitment flow of the study. CSI, corticosteroid injection; GRC, Global Rating of Change; NPRS, Numeric Pain Rating Scale; MPT, Manual Physical Therapy; SPADI, Shoulder Pain and Disability Index.

\section{Participants}

We will recruit 104 subjects, male and female, between the ages of 18 and 65 years with a primary complaint of shoulder pain through the Physical Therapy departments at Madigan Army Medical Center as well as the outlying primary care clinics on the military installation. They will be recruited through the referral source to Physical Therapy from outlying clinics (Family Practice, Orthopaedics and Primary Care clinics). If recruitment targets are not being met, flyers with information about the study may be distributed for potential subjects to enquire about, as patients in this setting have direct access to physical therapy care and do not need a referral source.

\section{Inclusion criteria:}

1. Age 18 and older.

2. Read, write, and speak English.

3. Eligible for healthcare at a military medical treatment facility.

4. Primary complaint of shoulder pain (glenohumeral region).

5. Meets diagnostic criteria for shoulder impingement (mentioned below).

\section{Exclusion criteria:}

1. History of a shoulder injection for the current episode of pain.

2. History of shoulder dislocation, subluxation, fracture, adhesive capsulitis the glenohumeral joint or cervical/shoulder/upper back surgery.
3. Isolated acromioclavicular joint (ACJ) pathology. The only location of symptoms is localised specifically with one finger directly over the ACJ and nowhere else, and reproduced only with ACJ palpation by the examiner.

4. Full-thickness rotator cuff tears (evidenced by MRI and/or positive lag signs).

5. Presence of cervical radiculopathy, radiculitis, or referral from cervical spine.

6. Total baseline SPADI score not $<20 \%$ (to prevent a ceiling effect with treatment).

7. History of breast cancer on the involved side.

8. Prior MPT treatment to the involved limb for the current episode of pain.

9. Military service members pending a medical evaluation board, physical evaluation board or equivalent discharge process, or in medical hold to determine long-term disposition. For non-military personnel, anyone who is pending or undergoing any litigation for their injury.

10. Contraindications or precautions to receiving a corticosteroid injection (history of allergies, adverse reactions, history of multiple injections in that area even if not within last 30 days, uncontrolled diabetes mellitus, pregnancy, etc).

11. Unable to give informed consent to participate in the study.

12. Unable to come into the clinic for regular treatment over the course of the following month.

\section{Diagnostic criteria for entry:}

To be included in the study, participants are required to have:

1. Pain with one of the two tests in category I; and

2. Pain with one test from either category II or category III.

with 'pain' being defined as reproduction of the usual pain that the subject experiences that makes up the nature of their complaint.

Category I: impingement signs:

1. Passive overpressure at full shoulder flexion with the scapula stabilised.

2. Passive internal rotation at $90^{\circ}$ of shoulder flexion in the scapular plane and in progressive degree of horizontal adduction.

Category II: active shoulder abduction:

Active shoulder abduction

Category III: resisted break tests:

1. Abduction

2. Internal rotation

3. External rotation

These criteria have been used in previous clinical trials to classify patients with shoulder impingement syndrome. ${ }^{17} 23$

\section{Randomisation}

Once the baseline examination is completed, a second investigator blind to the baseline examination will open the randomisation envelope indicating the 
patient's treatment group assignment that corresponds to the patient's unique identification number. A random-number generator will be used to establish randomisation lists prior to the initiation of the study. Individual randomisation assignments will be concealed according to the following procedure. The group assignment will be recorded on a label that is affixed to a $3.5 \times 5$ inch index card. This card will be folded in half such that the label with the patient's group assignment will be on the inside of the fold. The folded index card will then be placed inside the envelope, and the envelope will be sealed. This will prevent the possibility of the therapist holding the envelope up to the light and visualising the patient's treatment group assignment through a sealed yet transparent envelope.

\section{Blinding}

Owing to the nature of this study, it is not possible to blind the patient or the clinician providing the intervention to the treatment received. The clinician performing the screening, baseline and follow-up measurements, and outcome assessments will be blinded to the patient's treatment group assignment. Patients will be instructed not to discuss the intervention received with the clinician when reporting for their follow-up appointments unless medically necessary. Incidence of unmasking will be recorded.

\section{Interventions}

Both treatment options are standard-of-care interventions. Their allocation and dosage are described in table 1.

\section{Manual physical therapy}

At the first session, the examiner will perform a standard clinical examination in order to determine the appropriate application and delivery of MPT (appendix 1) to the entire shoulder, spine, and adjacent joints. The goal of MPT is to improve the quality of motion and decrease the pain associated with motion at the target joint and soft tissue. For our purposes, we define our MPT as the combination of manual techniques to include joint mobilisations (both thrust and non-thrust techniques), soft-tissue mobilisations, manual stretches and contract-relax techniques, along with the exercises that help reinforce those techniques. We feel this is consistent not only with other shoulder trials, but also with other MPT trials. ${ }^{9}{ }^{33-35}$ The application of techniques will not be based on a regimented protocol that is identical for each patient, but rather tailored specifically to the impairments identified during the clinical examination. We feel this approach is pragmatic and consistent with the actual delivery of MPT in the clinical setting.

The examiner will provide an initial intervention of MPT that is targeted to impairments found during the initial clinical examination (the patient will return for a maximum total of six treatment sessions over the following 3 weeks). Each session will last approximately $30 \mathrm{~min}$. The subjects will receive some of the protocol exercises (appendix 2) to augment the joint mobilisations, which will collectively make up the MPT intervention.

The clinicians providing the MPT are two fellowshiptrained orthopaedic manual physical therapists, who both received training in the same 18-month programme, to ensure consistency with delivery of this intervention approach.

\section{Corticosteroid injection}

The examiner will perform a standardised clinical examination and health screening in order to confirm the absence of contraindications to steroid injection. The subject will then receive an injection in the subacromial space of the symptomatic shoulder by a senior Sports Medicine Fellowship Trained Family Physician (appendix 3). The subject will also receive a handout explaining the effects of the steroid injection and how to manage any potential flare-ups or ensuing pain, and a handout describing pendulum exercises for the subject to perform. The physician will spend approximately 30 min with each subject explaining the rationale for the injection, relevant anatomy, performing the procedure and reviewing the pendulum exercises.

The subject can receive up to a total of two shoulder injections with a minimum of 1 month between injections if they are not getting relief of symptoms with the initial injection. This will be dictated by patient preference and the approval/recommendation of the physician performing the injection procedure, as this would represent a pragmatic approach commensurate with standard practice.

\section{Outcome measures}

The primary DV will be the SPADI, and the secondary DVs will be the GRC and the NPRS.

\section{Shoulder Pain and Disability Index}

SPADI is a 100-point, 13-item self-administered questionnaire, divided into two subscales: a five-item pain subscale and an eight-item disability subscale. Williams

Table 1 Treatment allocation and dosage

\begin{tabular}{|c|c|c|c|}
\hline Intervention & Subjects needed & Dosage & $\begin{array}{l}\text { Follow-up time points } \\
\text { (months) }\end{array}$ \\
\hline Corticosteroid injection & $\mathrm{N}=52$ & $\begin{array}{l}\text { One initially; option for a total } \\
\text { of three within first } 6 \text { months }\end{array}$ & $1,3,6,12$ \\
\hline Manual physical therapy & $\mathrm{N}=52$ & Six sessions & $1,3,6,12$ \\
\hline
\end{tabular}


et al have shown that the SPADI is responsive to change and accurately discriminates between patients who are improving or worsening. ${ }^{36}$ A high test-retest reliability and internal consistency for this instrument has been reported. ${ }^{37} \mathrm{~A}$ recent systematic review identified a minimal detectable change of 18 points and a minimally clinically important difference (MCID) of 8-13 points. ${ }^{38}$ The validity and responsiveness to change of SPADI have been described in physical therapy, as well as primary- and secondary-care settings. ${ }^{39}$

Because disability will be used as an outcome of interest, it is important to ensure a moderate level of disability will be present at the inception of the treatment. Thus, patients will be required to have at least a baseline SPADI score of 20 points (average of pain and disability subscales). A score of 0 indicates no pain or functional limitation with the described activities.

\section{Global Rating of Change questionnaire}

The GRC questionnaire is an instrument that measures overall changes in the quality of life of the subject. ${ }^{40}$ The use of a GRC is a common, feasible and useful method for assessing outcome, ${ }^{41}$ and has been shown to be a valid measurement of change in patient status in other pain populations. ${ }^{42}$ A change in score of three rating points $(+3)$ has been established as clinically significant in the patients perception of quality of life. ${ }^{41}$ The GRC has 15 possible choices, with 0 being equal to no change, -1 to -7 indicating a negative change and +1 to +7 indicating a positive change.

\section{Numeric Pain-Rating Scale}

An 11-point pain-rating scale ranging from 0 (no pain) to 10 (worst imaginable pain) will be used to assess pain intensity in the shoulder. ${ }^{43}$ This scale has been demonstrated to be reliable and generalisable, and to have internal consistency in measures of clinical and experimental pain sensation intensity. ${ }^{44} 45$ A change of at least three points has been suggested as the MCID for the NPRS in patients with shoulder pain. ${ }^{46}$

\section{Justification of sample size}

The calculations were based on detecting a 12-point (or $9.2 \%$ change - based on reported MCID range of 8-13 points) ${ }^{38}$ difference in the SPADI with a standard deviation of 10 points, a two-tailed test and an $\alpha$ level $=0.05$. This generates a sample size of 43 subjects per group. Allowing for a conservative dropout or loss to follow-up rate of approximately $20 \%$, we will recruit 104 subjects into the study. Planning for a larger loss to follow-up rate is deemed necessary in this military population where follow-up at 1 year can be challenging with multiple deployments and changes in duty station around the world. This sample size will yield greater than $80 \%$ power to detect both statistically significant and clinically meaningful changes in the other outcome variables. Sample-size estimation was performed with G*Power software, V 3.1.2. ${ }^{47}$

\section{Data analysis}

Descriptive statistics will be run on the demographic data (age, gender, race, etc) and health characteristics of the study population to include measures of central tendency (means, medians other percentiles) and dispersion (standard deviations, ranges) which will be computed for continuous data for summary. Frequency distributions will be estimated for categorical data. Graphical displays including histograms and box plots will be produced. Transformations will be sought for variables to be included in further analyses to ensure distributional assumptions are met.

We will examine the primary aim using a linear mixed model with repeated measures to account for the correlation among repeated observations from the same patient. Time (baseline, 1, 3, 6 and 12 months) and treatment group (MPT and CSI) will be modelled as fixed effects, with the SPADI questionnaire as the primary dependent variable. A separate model will be constructed in a similar fashion with pain (NPRS) and the GRC as the DV. The hypothesis of interest will be the group-by-time interaction. Treatment effects will be calculated from the between-group differences in change score from baseline to 1, 3, 6 and 12 months. No patients will be removed from the analysis for lack of adherence to treatment procedures. Missing data points will be estimated in the mixed model analyses using restricted maximum likelihood ratio estimation with 100 iterations. If one treatment is shown to be superior to the other, supplemental analyses will be performed by dichotomising groups based on minimal clinically important differences of $13 \%$ for the SPADI. ${ }^{45}$ This will allow computation of absolute risk reduction, relative risk reduction and number needed to treat (with associated $95 \%$ CIs) using failure to obtain clinically meaningful benefit as the event of interest. The level of significance for all analyses will be a priori established at 0.05 using a two-tailed test.

Because of potential crossover between groups, data will be analysed using both per-protocol and intentionto-treat principles. The per-protocol analyses will only include patients who completed the study in the group to which they were randomly assigned. The intention-totreat analyses will include all patients who were randomised at baseline regardless if they crossed over. Data analysis will be conducted using SPSS for Windows V 16.

\section{Trial organisation and monitoring}

The investigative team includes the authors listed in this protocol, in addition to three other licensed physical therapists who will assist with subject screening, data collection, subject follow-up and data entry. The principal investigator will manage data flow and perform audits of the procedures, enrolment and treatment throughout the entire process of the study. The associate investigators will monitor the data-collection process and data integrity with periodic evaluation performed continually during the course of the data-collection phase. 


\section{DISCUSSION}

This randomised clinical trial will be the first study that directly compares the short- and long-term effects of an impairment-based MPT approach and CSI for patients with shoulder impingement syndrome. The results of this study may help to establish best clinical practice guidelines for this patient population.

\section{ETHICAL CONSIDERATIONS AND DISSEMINATION}

All interventions provided in this study are considered standard of care and could be given to a patient as part of their treatment plan even if they were not part of this study. An ethics review will be conducted by Madigan Army Medical Center and monitored by the US Army Medical Department Clinical Investigation Regulatory Office to ensure compliance with federal regulations for protection of human medical research subjects. This clinical trial was registered with http:// clinicaltrials.gov/ with a registration number of NCT01190891.

\section{PUBLICATION POLICY}

The results of the trial will be published in an appropriate journal regardless of outcome. We will report the results following the CONSORT statement with the recommended extension for pragmatic trials. ${ }^{31}$

\section{PROJECTED TIMETABLE FOR TRIAL}

March 2010-protocol approved by the Western Regional Medical Command Institutional Review Board.

June 2010-subject enrolment begins.

June 2011-first subject completes 1-year follow-up.

July 2011-subject enrolment complete.

July 2012-last subject completes 1-year follow-up.

December 2012-data entry and analysis complete.

June 2013-publication with study results submitted for publication.

\section{Author affiliations:}

${ }^{1}$ Department of Physical Medicine, Madigan Army Medical Center, Tacoma, Washington, USA

${ }^{2}$ School of Physical Therapy, University of Puget Sound, Tacoma, Washington, USA

${ }^{3}$ School of Physical Therapy, Franklin Pierce University,Concord, New Hampshire, USA

${ }^{4}$ Department of Family Medicine, Madigan Army Medical Center, Tacoma, Washington, USA

Correction notice The "To cite: ..." information and running footer in this article have been updated with the correct volume number (volume 1).

Funding This study is funded in part by the Orthopaedic Physical Therapy Products Grant through the American Academy of Orthopaedic Manual Physical Therapists.

Competing interests None.

Ethics approval Ethics approval was provided by the Madigan Army Medical Center Institutional Review Board.

Contributions DIR conceived the idea for the project and is the PI. All authors contributed to writing and reviewing the protocol, as well as reviewing and submitting the protocol for publication. JAC provided advice with statistical and methods design. DIR and REB will provide all the direct interventions for the MPT group. DLB will perform all the injections for the CSI group.

Provenance and peer review Not commissioned; externally peer reviewed.

\section{REFERENCES}

1. Conroy DE, Hayes KW. The effect of joint mobilization as a component of comprehensive treatment for primary shoulder impingement syndrome. J Orthop Sports Phys Ther 1998;28:3-14.

2. Warner JJ, Micheli LJ, Arslanian LE, et al. Patterns of flexibility, laxity, and strength in normal shoulders and shoulders with instability and impingement. Am J Sports Med 1990;18:366-75.

3. Pope DP, Croft PR, Pritchard CM, et al. Prevalence of shoulder pain in the community: the influence of case definition. Ann Rheum Dis 1997:56:308-12.

4. Bot SD, van der Waal JM, Terwee CB, et al. Predictors of outcome in neck and shoulder symptoms: a cohort study in general practice. Spine (Phila Pa 1976) 2005:30:E459-70.

5. Croft P, Pope D, Silman A. The clinical course of shoulder pain: prospective cohort study in primary care. Primary Care Rheumatology Society Shoulder Study Group. BMJ 1996;313:601-2.

6. van der Windt DA, Koes BW, Boeke AJ, et al. Shoulder disorders in general practice: prognostic indicators of outcome. $\mathrm{Br} J$ Gen Pract 1996;46:519-23.

7. Winters JC, Jorritsma W, Groenier KH, et al. Treatment of shoulder complaints in general practice: long term results of a randomised, single blind study comparing physiotherapy, manipulation, and corticosteroid injection. BMJ 1999;318:1395-6.

8. Winters JC, Sobel JS, Groenier KH, et al. The long-term course of shoulder complaints: a prospective study in general practice. Rheumatology (Oxford) 1999:38:160-3.

9. Bang MD, Deyle GD. Comparison of supervised exercise with and without manual physical therapy for patients with shoulder impingement syndrome. J Orthop Sports Phys Ther 2000;30:126-37.

10. Bergman GJ, Winters JC, Groenier KH, et al. Manipulative therapy in addition to usual medical care for patients with shoulder dysfunction and pain: a randomized, controlled trial. Ann Intern Med 2004;141:432-9.

11. Winters JC, Sobel JS, Groenier KH, et al. Comparison of physiotherapy, manipulation, and corticosteroid injection for treating shoulder complaints in general practice: randomised, single blind study. BMJ 1997;314:1320-5.

12. Boyles RE, Ritland BM, Miracle BM, et al. The short-term effects of thoracic spine thrust manipulation on patients with shoulder impingement syndrome. Man Ther 2009;14:375-80.

13. Galgano RC. Manipulative therapy for patients with shoulder symptoms. Ann Intern Med 2005;142:226; author reply 26-7.

14. Haddick E. Management of a patient with shoulder pain and disability: a manual physical therapy approach addressing impairments of the cervical spine and upper limb neural tissue. J Orthop Sports Phys Ther 2007;37:342-50.

15. Kachingwe AF, Phillips B, Sletten E, et al. Comparison of manual therapy techniques with therapeutic exercise in the treatment of shoulder impingement: a randomized controlled pilot clinical trial. $J$ Man Manip Ther 2008;16:238-47.

16. Senbursa G, Baltaci G, Atay A. Comparison of conservative treatment with and without manual physical therapy for patients with shoulder impingement syndrome: a prospective, randomized clinical trial. Knee Surg Sports Traumatol Arthrosc 2007;15:915-21.

17. Ho CY, Sole G, Munn J. The effectiveness of manual therapy in the management of musculoskeletal disorders of the shoulder: a systematic review. Man Ther 2009;14:463-74.

18. Yiasemides R, Halaki M, Cathers I, et al. Does passive mobilization of shoulder region joints provide additional benefit over advice and exercise alone for people who have shoulder pain and minimal movement restriction? A randomized controlled trial. Phys Ther 2011:91:178-89.

19. Chen JF, Ginn KA, Herbert RD. Passive mobilisation of shoulder region joints plus advice and exercise does not reduce pain and disability more than advice and exercise alone: a randomised trial. Aust J Physiother 2009;55:17-23.

20. Gruson KI, Ruchelsman DE, Zuckerman JD. Subacromial corticosteroid injections. J Shoulder Elbow Surg 2008;17(1 Suppl):118S-30S.

21. Alvarez CM, Litchfield R, Jackowski D, et al. A prospective, doubleblind, randomized clinical trial comparing subacromial injection of betamethasone and xylocaine to xylocaine alone in chronic rotator cuff tendinosis. Am J Sports Med 2005;33:255-62.

22. Akgun K, Birtane M, Akarirmak U. Is local subacromial corticosteroid injection beneficial in subacromial impingement syndrome? Clin Rheumatol 2004;23:496-500.

23. Arroll B, Goodyear-Smith F. Corticosteroid injections for painful shoulder: a meta-analysis. Br J Gen Pract 2005;55:224-8. 
24. Buchbinder R, Green S, Youd JM. Corticosteroid injections for shoulder pain. Cochrane Database Syst Rev 2003;(1):CD004016.

25. Cummins CA, Sasso LM, Nicholson D. Impingement syndrome: temporal outcomes of nonoperative treatment. J Shoulder Elbow Surg 2009;18:172-7.

26. Gaujoux-Viala C, Dougados M, Gossec L. Efficacy and safety of steroid injections for shoulder and elbow tendonitis: a meta-analysis of randomized controlled trials. Ann Rheum Dis 2009;68:1843-9.

27. Koester MC, Dunn WR, Kuhn JE, et al. The efficacy of subacromia corticosteroid injection in the treatment of rotator cuff disease: A systematic review. J Am Acad Orthop Surg 2007;15:3-11.

28. Adebajo AO, Nash P, Hazleman BL. A prospective double blind dummy placebo controlled study comparing triamcinolone hexacetonide injection with oral diclofenac $50 \mathrm{mg}$ TDS in patients with rotator cuff tendinitis. J Rheumatol 1990;17:1207-10.

29. Henkus HE, Cobben LP, Coerkamp EG, et al. The accuracy of subacromial injections: a prospective randomized magnetic resonance imaging study. Arthroscopy 2006;22:277-82.

30. Borick JM, Kurzweil PR. Magnetic resonance imaging appearance of the shoulder after subacromial injection with corticosteroids can mimic a rotator cuff tear. Arthroscopy 2008;24:846-9.

31. Zwarenstein M, Treweek S, Gagnier JJ, et al. Improving the reporting of pragmatic trials: an extension of the CONSORT statement. BMJ 2008;337:a2390.

32. Anon. Strengthening the credibility of clinical research. Lancet 2010;375:1225.

33. Deyle GD, Allison SC, Matekel RL, et al. Physical therapy treatment effectiveness for osteoarthritis of the knee: a randomized comparison of supervised clinical exercise and manual therapy procedures versus a home exercise program. Phys Ther 2005;85:1301-17.

34. Deyle GD, Henderson NE, Matekel RL, et al. Effectiveness of manual physical therapy and exercise in osteoarthritis of the knee. A randomized, controlled trial. Ann Intern Med 2000;132:173-81.

35. Hoeksma HL, Dekker J, Ronday HK, et al. Comparison of manual therapy and exercise therapy in osteoarthritis of the hip: a randomized clinical trial. Arthritis Rheum 2004:51:722-9.
36. Williams JW Jr, Holleman DR Jr, Simel DL. Measuring shoulder function with the Shoulder Pain and Disability Index. J Rheumatol 1995;22:727-32.

37. Michener LA, Leggin BG. A review of self-report scales for the assessment of functional limitation and disability of the shoulder. $J$ Hand Ther 2001;14:68-76.

38. Roy JS, MacDermid JC, Woodhouse LJ. Measuring shoulder function: a systematic review of four questionnaires. Arthritis Rheum 2009;61:623-32.

39. Roach KE, Budiman-Mak E, Songsiridej N, et al. Development of a shoulder pain and disability index. Arthritis Care Res 1991;4:143-9.

40. Juniper EF, Guyatt GH, Willan A, et al. Determining a minimal important change in a disease-specific Quality of Life Questionnaire. J Clin Epidemiol 1994;47:81-7.

41. Jaeschke R, Singer J, Guyatt GH. Measurement of health status. Ascertaining the minimal clinically important difference. Control Clin Trials 1989:10:407-15.

42. Fritz JM, Irrgang JJ. A comparison of a modified Oswestry Low Back Pain Disability Questionnaire and the Quebec Back Pain Disability Scale. Phys Ther 2001;81:776-88.

43. Jensen MP, Turner JA, Romano JM. What is the maximum number of levels needed in pain intensity measurement? Pain 1994:58:387-92.

44. Jensen MP, McFarland CA. Increasing the reliability and validity of pain intensity measurement in chronic pain patients. Pain 1993:55:195-203.

45. Price DD, Bush FM, Long S, et al. A comparison of pain measurement characteristics of mechanical visual analogue and simple numerical rating scales. Pain 1994;56:217-26.

46. Michener LA, Snyder AR, Leggin BG. Responsiveness of the numeric pain rating scale in patients with shoulder pain and the effect of surgical status. J Sport Rehabil 2011;20:115-28.

47. Faul F La-G BA. G* Power. In

48. Rhon D, Hando B. Evidence Based OMT $1.1 \mathrm{ed}$. Available at http:// itunes.apple.com/us/app/evidence-based-omt/id414629908? $\mathrm{mt}=12$ (accessed 22 Mar 2011). 


\section{APPENDIX 1}

Manual physical therapy manual techniques. ${ }^{48}$

Represents the majority of the techniques used, but is not inclusive or representative of all of them.

\section{Manual Physical Therapy Techniques ${ }^{48}$}

\section{Glenohumeral Joint}

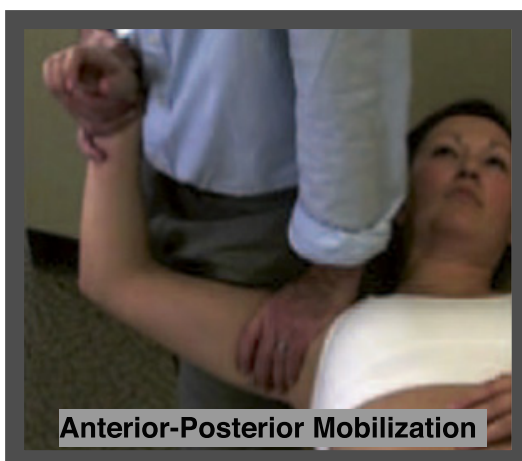

Clinician Position:

Grasp patient's wrist and stabilize arm against trunk. Heel/lateral border of mobilizing hand is on anterior joint.

Technique Description:

Apply a graded mobilization into a posterior direction. Abduction can vary.

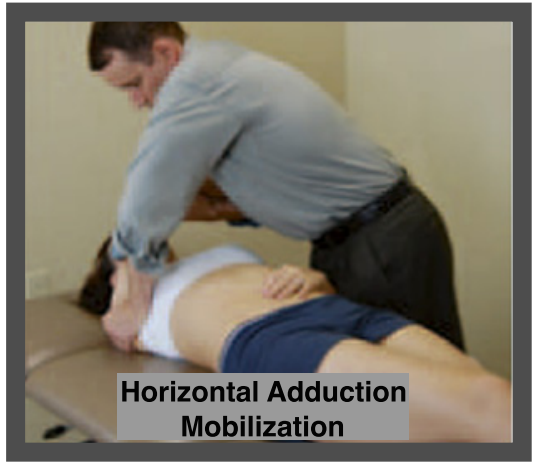

Clinician Position:

Position heel of caudal hand on lateral border of scapula and provide firm stabilizing force.

Technique Description:

With the scapula stabilized, move the humerus into horizontal adduction with cephalad hand.

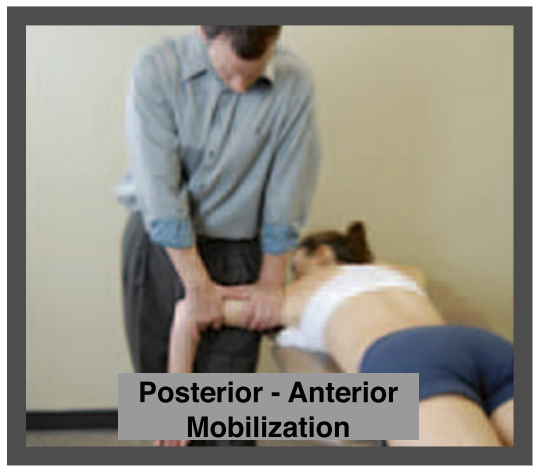

Clinician Position:

Support the distal humerus and elbow. The heel of the other hand is placed over posterior glenohumeral head.

Technique Description:

A posterior to anterior force is imparted through the locked elbow.

Glenohumeral abduction can be varied.

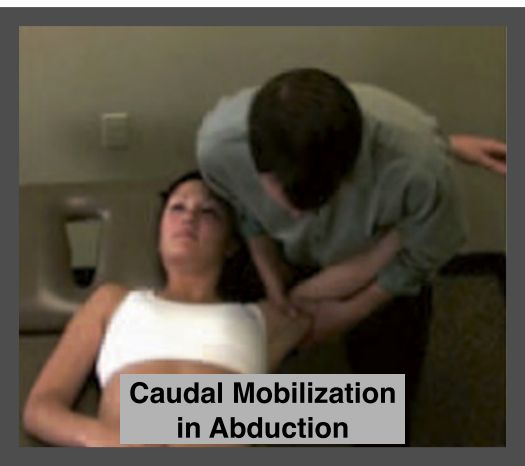

\section{Clinician Position:}

Grasp patient's glenohumeral joint with both hands. Stabilize arm against body. Technique Description:

Apply a graded mobilization into a caudal/inferior direction. Abduction can vary.

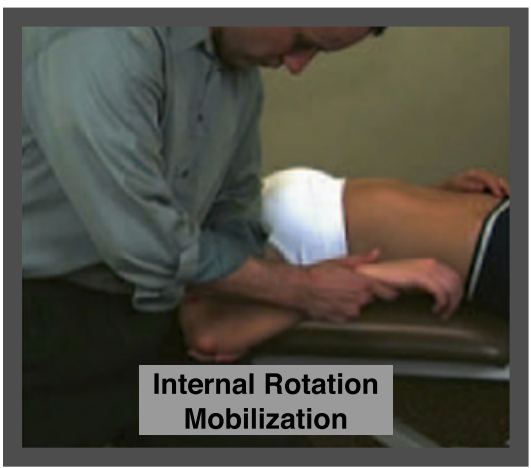

Clinician Position:

Proximal forearm stabilizes the patient's with their elbow over the clavicle. Gently grasp the patient's wrist.

Technique Description:

A rotatory internal rotation force is imparted on the humerus while proxima arm provides stabilization.

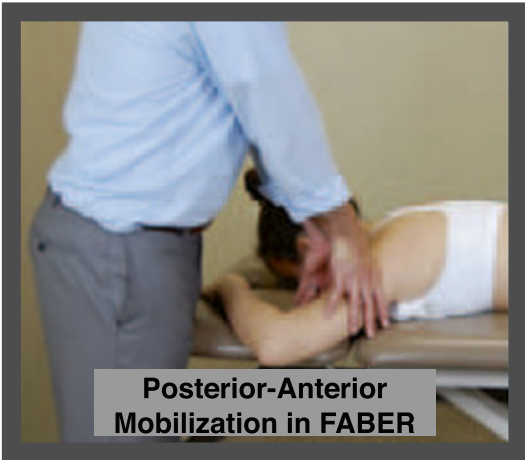

\section{Clinician Position:}

The joint is grasped by both hands with the thumbs and webspaces of the hands on the superior aspect and the fingers cupped underneath.

Technique Description:

With elbows locked, a posterior to anterior force is imparted.

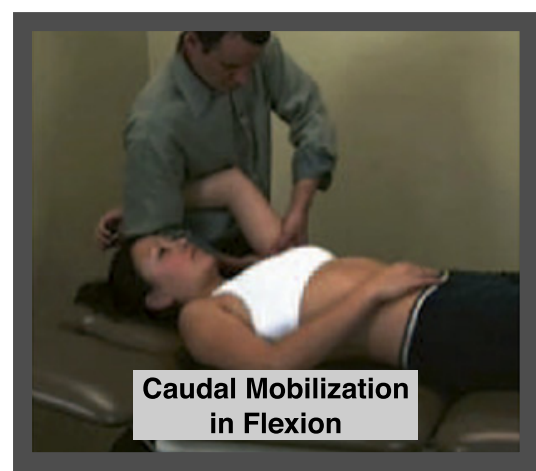

Clinician Position:

Grasp patient's glenohumeral joint with both hands. Stabilize arm against body. Technique Description:

Apply a graded mobilization into a caudal/inferior direction. Flexion can vary.

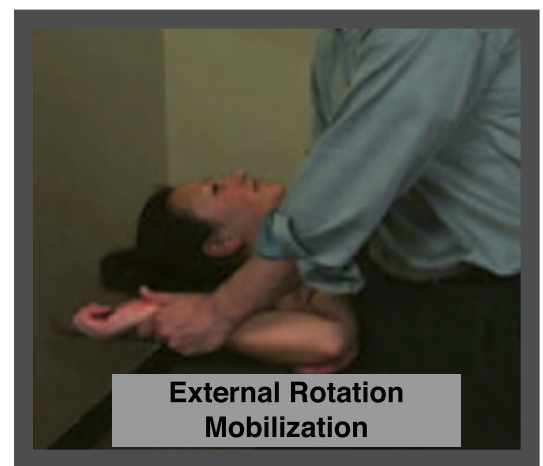

Clinician Position:

Proximal forearm stabilizes the patient's with their elbow over the clavicle. Gently grasp the patient's wrist.

Technique Description:

A rotatory external rotation force is imparted on the humerus while proximal arm provides stabilization.

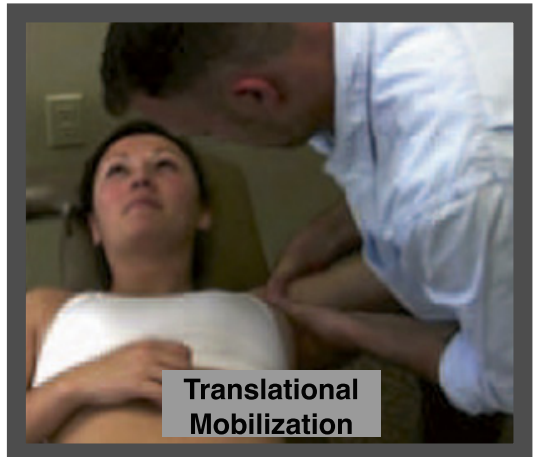

Clinician Position:

Stand to side of patient and support their distal arm up against your side. Both hands grasp the joint.

Technique Description:

A short lever-arm translational glide is imparted at the glenohumeral joint in anterior, posterior, inferior, and superior directions. 


\section{Orthopaedic Manual Physical Therapy Techniques 48}

\section{Acromioclavicular Joint}

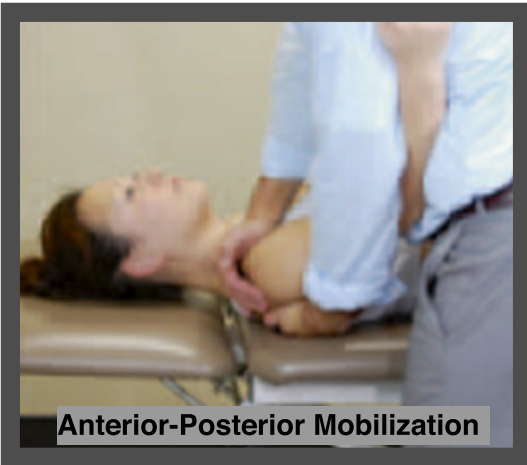

Clinician Position:

One hand underneath the shoulder to stabilize the scapula. The pisiform is gently applied to the distal clavicle Technique Description:

Use the pisiform to apply a caudallydirected mobilization.

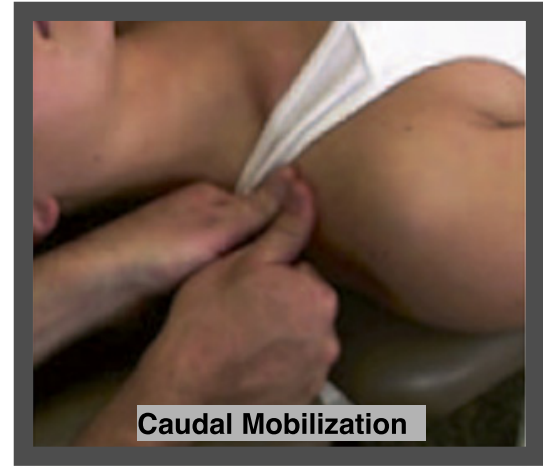

\section{Clinician Position:}

The tips of both thumbs on the cephalad surface of the distal clavicle and place thumbs side by side for stability.

Technique Description:

Force is applied through the arms and thumbs caudally into the distal acromion.

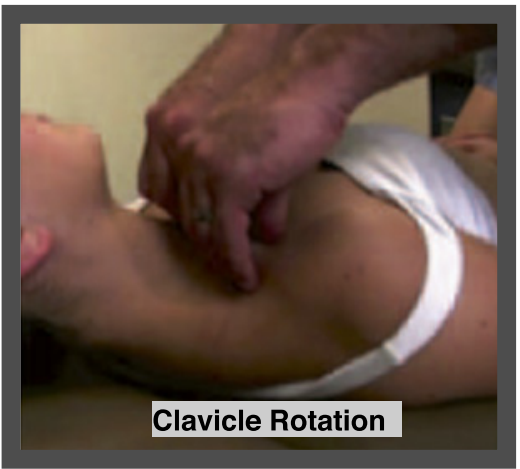

Clinician Position:

Gently grip the middle of the clavicle. Technique Description:

Apply a gentle force using a rocking or "wiggling" motion through repetitive wrist flexion and extension to impart rotation of the clavicle on its long axis.

\section{Thoracic Spine and Scapular-Thoracic Joint}

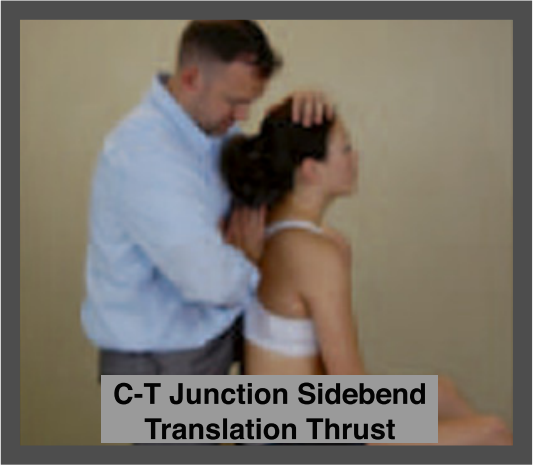

Clinician Position:

Gently place contralateral hand on the patient's head, with knee blocking the lateral trunk.

Technique Description:Using your pisiform on the spinous process, impart a progressive translatory force.

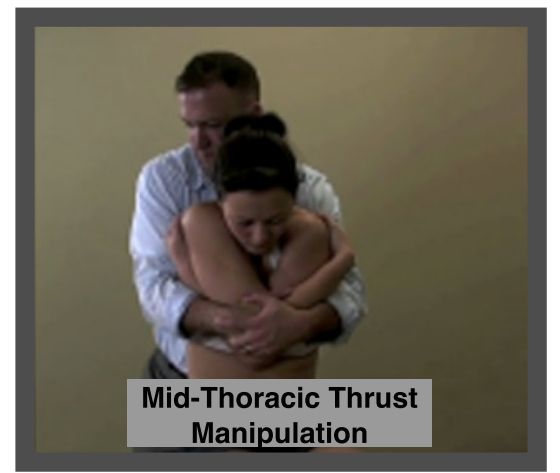

Clinician Position:

Arms around patient clasped around the elbows. Place towel as fulcrum at designated level.

Technique Description:

Fulcrum the thoracic spine into slight flexion. Take up restriction. Impart force by extending knee \& lifting vertically.

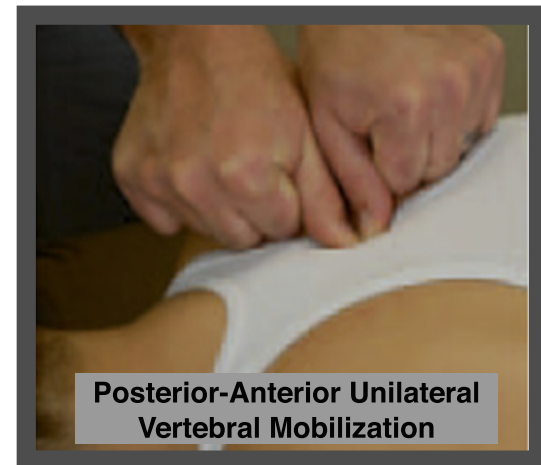

\section{Clinician Position:}

Stand to the side of the patient. Technique Description: Force is applied from trunk/shoulders through the arms and thumbs to the articular pillar.

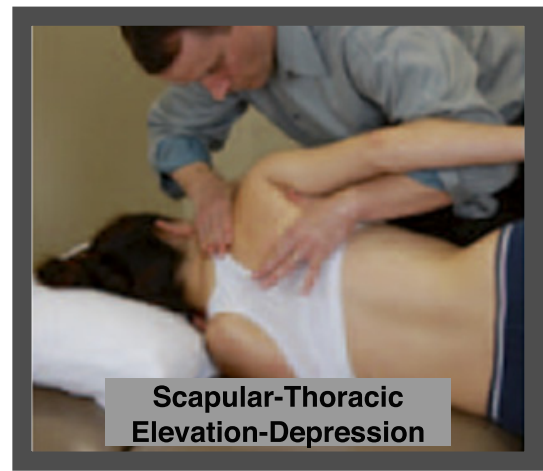

Clinician Position:

Grasp the inferior angle. and spine of the scapula

Technique Description:

The motion comes from the clinician's trunk to mobilize the scapula in a cephalad and caudal direction.

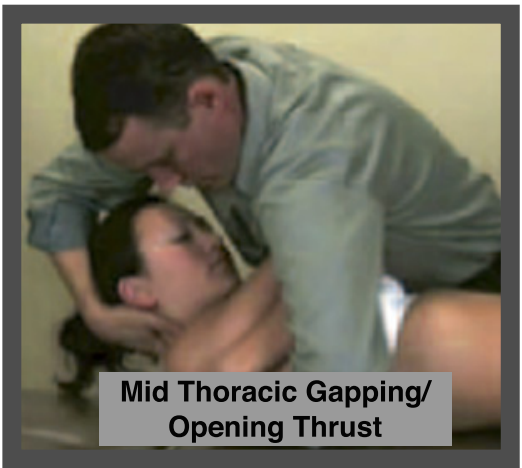

Clinician Position:

Stand on contralateral side of patient Technique Description:

Thenar eminence is on the transverse process. Establish skin lock with ulnar deviation of wrist. Support head with other am. Take up restriction and thrust anterior to posterior

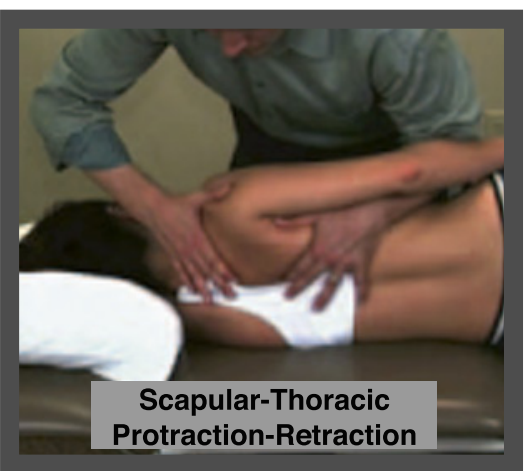

Clinician Position:

Grasp the inferior angle. and spine of the scapula

Technique Description:

The motion comes from the clinicians trunk to move the scapula laterally to produce protraction and medial to produce retraction. 


\section{APPENDIX 2}

Home exercise programme handout for MPT group

\section{WAND RANGE OF MOTION EXERCISES}

Lay on your back to stabilize your shoulder blade and place the hand of your painful shoulder over the wand and with the opposite arm push the wand so that your painful arm goes up overhead. Try not to bend your elbow. Do it straight and out to the side as well. Hold at end of range for 3 seconds.

30 Repetitions 2-3x/day

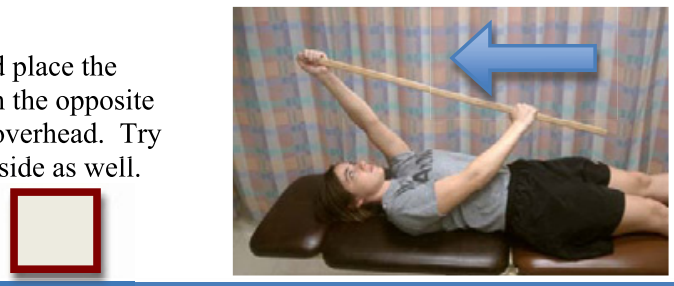

HOME EXERCISE PROGRAM
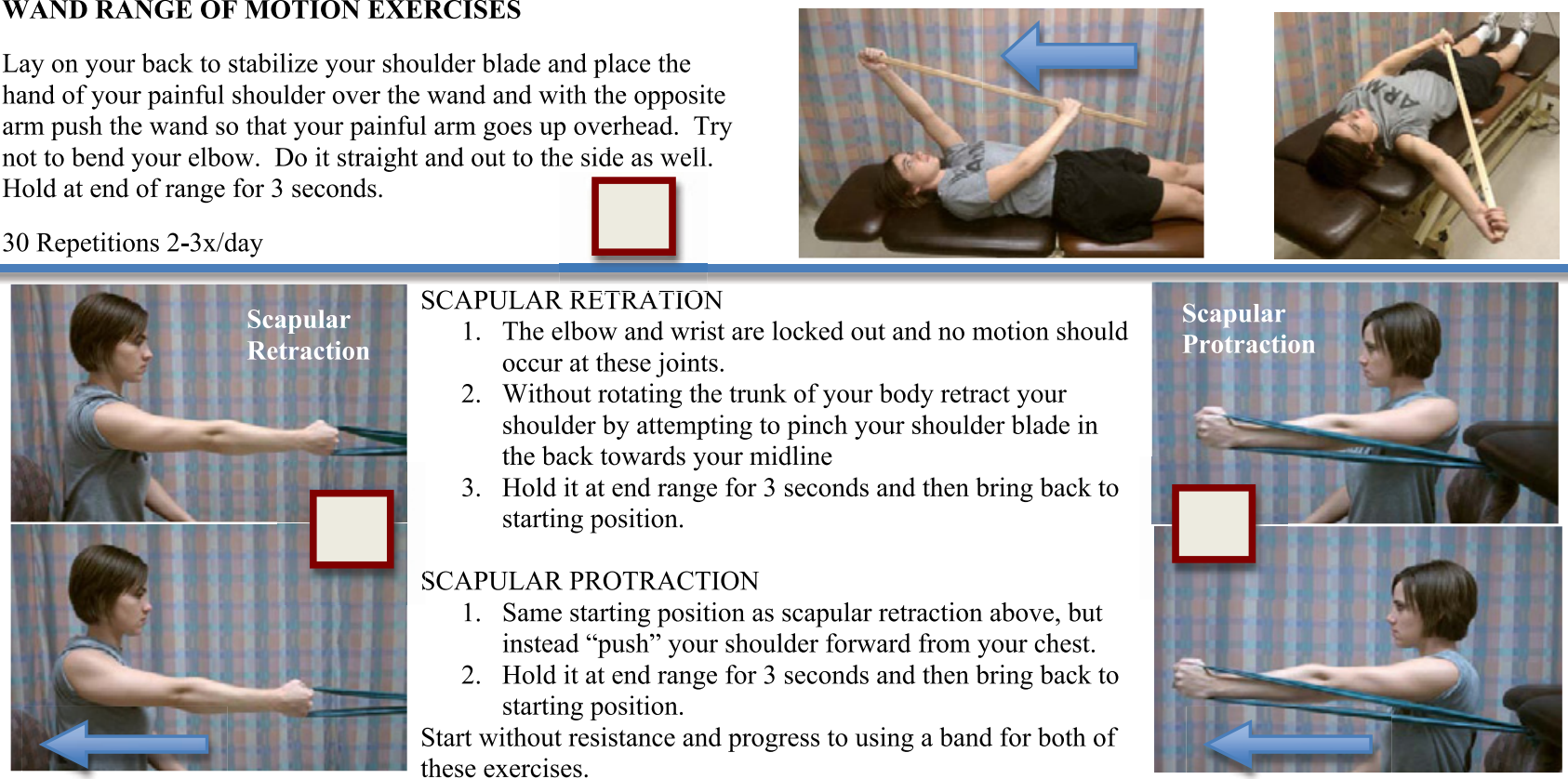

SCAPULAR RETRATIOON

1. The elbow and wrist are locked out and no motion should occur at these joints.

2. Without rotating the trunk of your body retract your shoulder by attempting to pinch your shoulder blade in the back towards your midline

3. Hold it at end range for 3 seconds and then bring back to starting position.

\section{SCAPULAR PROTRACTION}

1. Same starting position as scapular retraction above, but instead "push" your shoulder forward from your chest.

2. Hold it at end range for 3 seconds and then bring back to starting position.

Start without resistance and progress to using a band for both of these exercises.

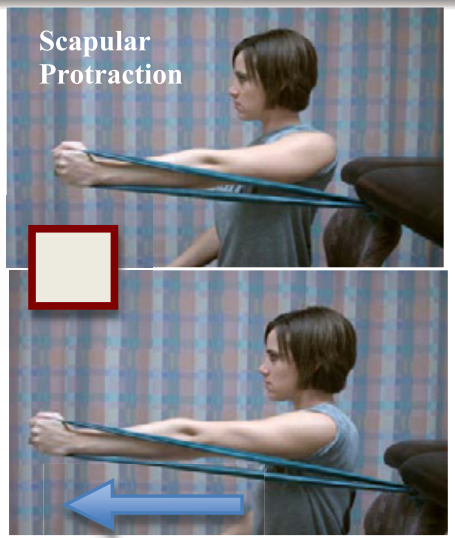

$3 \times 10$ Repetitions 2-3x/day

\section{THORACIC SELF-MOBILIZATION}

1. Place a rolled up towel with the top edge at the segment where you want to create a fulcrum and mobilize

2. Interlock your fingers on your neck behind your head

3. Bring your elbows together so that they are point straight out in front of you.

4. Extend backwards to point your elbows up towards the ceiling.

5. Return to the starting position.

3 sets of 30 repetitions (2-3x/day)
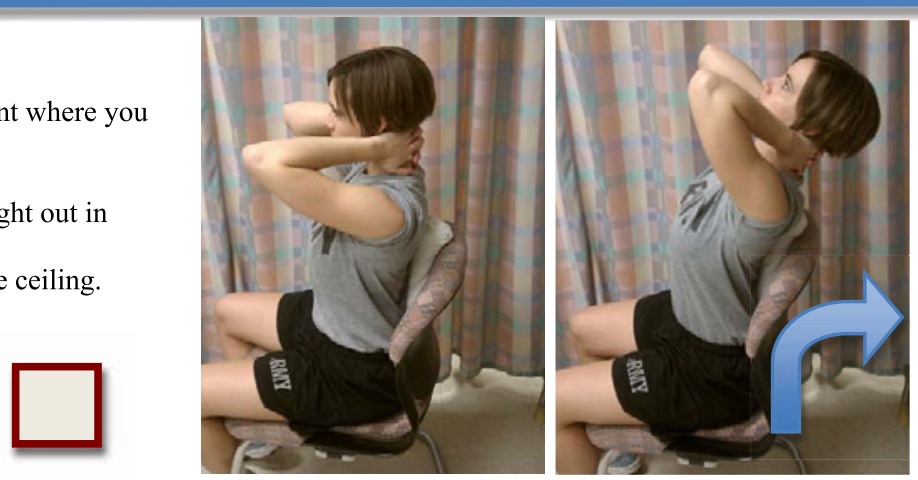

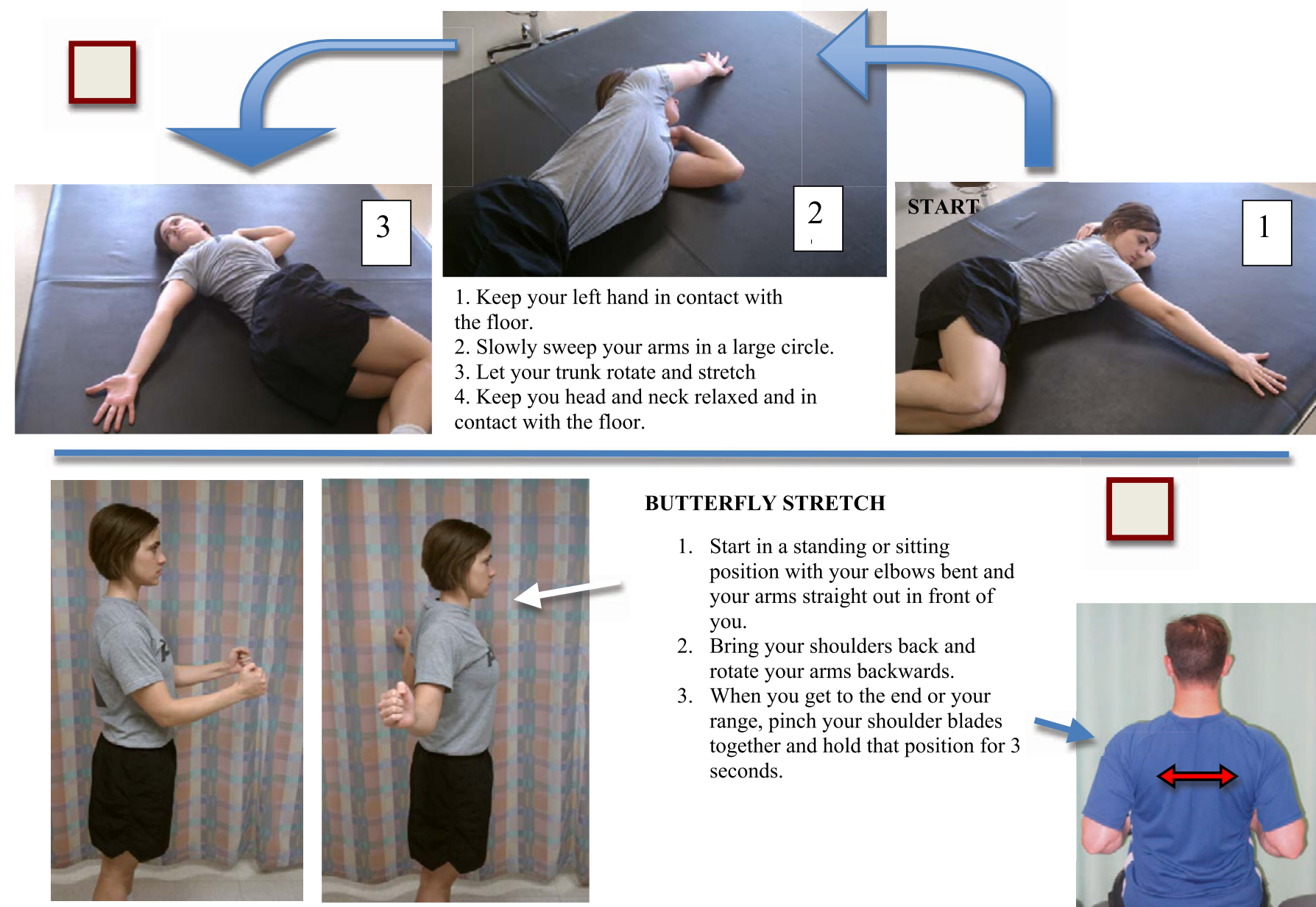

\section{BUTTERFLY STRETCH}

1. Start in a standing or sitting position with your elbows bent and your arms straight out in front of you.

2. Bring your shoulders back and rotate your arms backwards.

3. When you get to the end or your range, pinch your shoulder blades together and hold that position for 3 seconds.

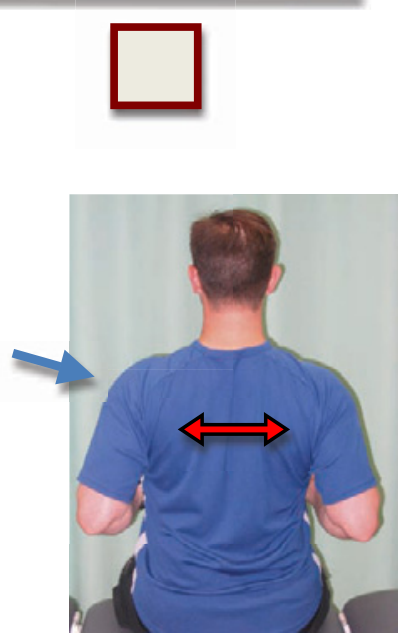




\section{APPENDIX 3}

Corticosteroid injection procedures

\section{Injection Procedures}

The clinician administering the injection using the technique described below. Sterile technique and precautions will be utilized. All standard care precautions will be utilized to include aspirating before applying the dose of medication to ensure that the needle has not been placed into a blood vessel. Patients will remain in the clinic for at least thirty minutes after the procedure to ensure there are no adverse effects. They will be educated regarding potential steroid flare, which can occur within the first 48 hours and can be treated with ice. Dosage will remain between 40-80 mg per injection, based on most current recommendations (Skedros 2007)

The following dose represents a glucocorticoid potency of 400 hydrocortisone equivalents/injection (mg).

Location: $\quad$ Subacromial space

Syringe: $\quad 10 \mathrm{~mL}$

Needle: $\quad 25$ gauge, 1.5 inch

Anesthetic: $\quad 6 \mathrm{~mL}$ of $0.5 \%$ Marcaine

Corticosteroid: $\quad 1.0-2.0 \mathrm{~mL}$ Triamcinolone Acetonide (Kenalog), $40 \mathrm{mg} / \mathrm{mL}$

\section{TECHNIQUE:}

The distal, lateral, and posterior edges of the acromion are palpated. Using aseptic technique, the needle is inserted just inferior to the posterolateral edge of the acromion. The needle is directed toward the opposite nipple. The pharmaceutical material should flow freely into the space without any resistance or significant discomfort to the patient. Followup care is the same as previously described.

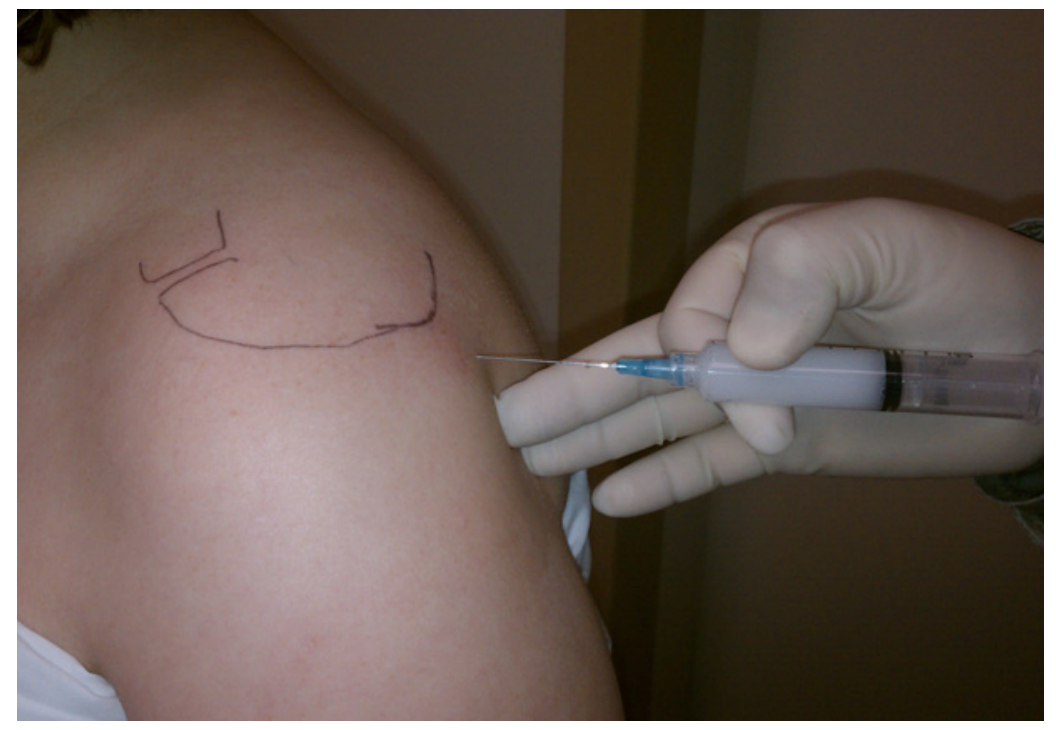

- Tallia, Diagnostic and Therapeutic Injection of the Shoulder Region, American Family Physician, 2003

- Skedros, Variations in corticosteroid/anesthetic injections for painful shoulder conditions : comparisons among orthopaedic surgeons, rheumatologists, and physical medicine and primary-care physicians. BMC Musculoskeletal Disorders, 2007 